\title{
REVISIÓN BIBLIOGRÁFICA RELACIÓN ENTRE LA ESCLEROSIS MÚLTIPLE Y LA CALIDAD DE VIDA
}

\author{
Daniela Gallego Erazo*, Luisa Fernanda Jiménez Jaramillo*, Paola Andrea López Gil*, David Fernando \\ López Rúales*, Stefany Marín Díaz*, María Mónica Peñaloza Beltrán*, Carmen Victoria Toro Rubiano* \\ Jheimy Jackeline García Castañeda**, Natalia Ramírez Girón***
*Estudiantes de Quinto Semestre de Enfermería, Universidad del Quindío
** Mg en Salud Pública -Docente del Programa de Enfermería, Universidad del Quindío
***Mg en Ciencias de Enfermería-Docente del Programa de Enfermería, Universidad del Quindío Correspondencia: Programa de enfermería, Universidad del Quindío - Av. Bolívar calle 12 Norte Arme- nia, Quindío - Colombia. E-mail: investigacion.5.2016@gmail.com - jheimyjacke@gmail.com - nrami- rez@uniquindio.edu.co

Correspondencia: Programa de enfermería, Universidad del Quindío - Av. Bolívar calle 12 Norte Armenia, Quindío - Colombia. E-mail: investigacion.5.2016@gmail.com - jheimyjacke@gmail.com - nramirez@uniquindio.edu.co

\begin{abstract}
RESUMEN
Introducción: La esclerosis múltiple (EM) es una enfermedad desmielinizante, inflamatoria y neurodegenerativa del sistema nervioso central, de etiología desconocida y patogenia autoinmune. Se estima que esta enfermedad afecta a 2,1 millones de personas a nivel mundial. Objetivo: Realizar un análisis descriptivo de la esclerosis múltiple y la manera en que esta afecta la calidad de vida, tanto de quien la presenta, como a su red de apoyo y/o cuidador principal. Metodología: Se realizó una revisión sistemática de tipo descriptivo, en la cual, se ejecutó a través de diferentes bases de datos. Se incluyeron artículos de investigación y revisión que estuvieran dentro de los años 2000-2015, publicados en idioma castellano. Resultados y discusión: Analizando las diversas fuentes consultadas, se encontraron grandes similitudes en los resultados de dichas investigaciones, entendiendo así que la EM es una enfermedad que afecta la calidad de vida de las personas en la misma manera, sin importar el lugar en el cual esta se desarrolle, teniendo en cuenta que las oportunidades para mejorar la calidad de vida de las personas después de un tratamiento, dependerá en gran medida del nivel de desarrollo en salud de la región en la que se presente el fenómeno. Conclusiones: La calidad de vida de las personas que presentan EM se ve afectada de manera drástica, ya que esta enfermedad puede llegar a ser completamente incapacitante en estadios avanzados, lo cual afecta no solo a la persona que la presenta sino también, su red de apoyo o cuidadores.Palabras claves: Voz hipernasal, labio y/o paladar hendido, Teager, TEO, MFCC, Pitch, Jitter, Shimmer, Formantes, Procesamiento digital de señales, electro-medicina.
\end{abstract}

Palabras clave: Esclerosis múltiple, calidad de vida, enfermería, discapacidad, autoinmune. 


\begin{abstract}
Introduction: Multiple sclerosis (MS) is a demyelinating, inflammatory and neurodegenerative disease of the central nervous system of unknown etiology and autoimmune pathogenesis. It is estimated that this disease affects 2.1 million people worldwide. Objective: To perform a descriptive analysis of multiple sclerosis and how this affects the quality of life, both of whom presented, and its support network and / or primary caregiver.

Methodology: A systematic review of descriptive, in which the documentary research was carried out through different databases was performed. research and review articles that were in the years 2000-2015, published in Castilian language were included. Results and discussion: Analyzing the various sources, great similarities in the results of those investigations were found, and understanding that MS is a disease that affects the quality of life of people in the same way, regardless of the place where this is developed, taking into account the opportunities to improve the quality of life of people after treatment, will depend largely on the level of health development in the region in which this phenomenon. Conclusions: The quality of life of people who have MS is affected drastically, as this disease can be complemented disabling in advanced stages, which affects not only the person who presents but also its network support or caregivers.
\end{abstract}

Keywords: multiple sclerosis, quality of life, nursing, disability, autoimmune.

\section{INTRODUCCIÓN}

La esclerosis múltiple (EM) es una enfermedad desmielinizante, inflamatoria y neurodegenerativa del sistema nervioso central (SNC), de etiología desconocida y patogenia autoinmune, caracterizada por la destrucción de la mielina, el fallo en su reparación y por un grado variable de lesión axonal. Se estima que esta enfermedad afecta a 2,1 millones de personas a nivel mundial (1), afectando principalmente al sexo femenino en edad reproductiva y de raza blanca entre edades de 20 a 40 años aproximadamente, la mayor prevalencia es en el hemisferio norte, países desarrollados y más aún en países como Estados Unidos y Canadá. Presentándose específicamente con mayor frecuencia en países del norte de Europa y Norteamérica (2). En Latinoamérica se señala los siguientes hallazgos: en México, se describió en 1970 una prevalencia de 1,6 casos por cada 100,000 habitantes, en Uruguay una prevalencia de 17,6 casos por cada 100,000 habitantes, en Brasil entre 4 a 15 casos por cada100.000 habitantes, en Argentina entre 15 a 18 casos por cada 100,000 habitantes(3). En Colombia la prevalencia de la esclerosis múltiple es de 1,48 a 4,98 casos por 100,000 habitantes aproximadamente, encontrándose la más elevada en el departamento de Risaralda con un valor de 4,98 por cada 100,000 habitantes y la más baja en Antioquia con 1,48 casos por cada 100,000 habitantes, coincidiendo con datos internacionales en los que se ve afectado principalmente el sexo femenino con un índice del 72\% (2). La EM afecta la calidad de vida tanto en quien la pre- senta como en su red de apoyo o cuidador, entendiéndose este concepto según la Organización Mundial de la Salud (OMS) como: "la percepción del individuo sobre una situación en la vida dentro del contexto cultural y de los valores en la que vive, y en la relación con sus objetivos, normas e inquietudes" (4). De igual manera esta está relacionada con la salud desde la perspectiva de que es un concepto complejo enel que se engloba la valoración de los dominios físicos, emocionales y sociales del individuo(5); diversos estudios han demostrado que la calidad de vida en personas con esclerosis múltiple se ve afectada significativamente a nivel personal, familiar, social, psicológico, cognitivo, laboral y económico, lo cual lleva al individuo a un declive de su estatus vital; asimismo, esta situación trae consigo implicaciones económicas mostrando así que a pesar de su baja prevalencia en la población, la EM tiene un impacto económico marcado. Para el periodo 2002-2005, la EM fue el diagnóstico que representó el mayor valor recobrado al Fondo de Solidaridad y Garantías (FOSYGA). Fondo para el manejo de recursos del SGSSS con un monto de cerca de $\$ 28$ mil millones de pesos, (cerca del $80 \%$ de lo que Colombia gastó en vacunas para el Programa Nacional de Inmunizaciones ese mismo año); no se conocen estudios del costo de la EM en Colombia aunque hay publicaciones asociadas (6). Además, requiere un manejo multidisciplinario aumentando el costo social. Igualmente se debe tener en cuenta el papel que desarrolla enfermería en el cuidado integral de la persona con EM, siendo de gran importancia debido a que contribuye al mejoramiento 
de la calidad de vida; por tanto la teoría de autocuidado de Dorothea Orem en 1971 explica el concepto de autocuidado como una contribución constante del individuo a su propia existencia: "El autocuidado es una actividad aprendida por los individuos, orientada hacia un objetivo. Es una conducta que existe en situaciones concretas de la vida, dirigida por las personas sobre sí mismas hacia los demás o hacia el entorno, para regular los factores que afectan a su propio desarrollo y funcionamiento en beneficio de su vida, salud o bienestar"(7). teniendo en cuenta que la disciplina de enfermería se involucra en el cuidado necesario para las personas con dicha enfermedad, en ocasiones sin intención de daño se omiten los principios de autonomía, acción que provoca el paternalismo, el cual se define como la limitación intencional de la autonomía de otra persona justificada por una apelación a la beneficencia, el bienestar o necesidades de otros(8), sin dejar de lado que el manejo de la EM es multidisciplinaria y que la enfermería es una profesión holística que siempre mira más allá de lo que no se puede ver, entendiéndose la función de enfermería como brindar servicios y cuidados que buscan mantener la salud, la vida y el bienestar, según sus requerimientos y de acuerdo a sus situaciones personales.

Por ende, en momentos son las fuentes de apoyo o red de apoyo de las personas que cursan por este proceso y tal como Dorothea Orem(7) plantea en su teoría y relacionándolo con el caso de las personas que desarrollan esta enfermedad son seres humanos realistas frente a sus capacidades de autocuidado y de los obstáculos que se le presentan; quien a como consecuencia de su patología, llegan a perder la capacidad de llevar a cabo acciones de autocuidado, pero siempre son conscientes de las mismas, por esto en su salud hay un deterioro de la integridad física, estructural y funcional; deterioro de la persona, su salud y bienestar tanto físico como psicológico y emocional, afectando la calidad de vida de la persona, el entorno juega un papel importante en las personas con EM porque se ve alterado principalmente por su limitación física, ya que el espacio en donde se pueden desenvolver se reduce significativamente por las condiciones de los lugares, viéndose afectado también a nivel social por la deserción laboral además; al volverse completamente dependientes necesitan de un cuidador permanente, lo cual genera una sobrecarga perjudicando su relaciones sociales, vida laboral, salud física y emocional, entre otros aspectos; dada esta situación he allí la importancia de implementar y conocer cómo manejar la situación utilizando diversas herramientas que ayuda- rán a brindar un cuidado sin dejar de lado ningún aspecto de la persona, no solo por respeto así la misma, sino que esto también afecta a los que se encuentran a su alrededor; una de las cosas más comunes que se puede encontrar en estos casos, es el síndrome de carga del cuidador, como se ha mencionado anteriormente, se afectan varios campos en la persona, entre esas la familia y quizás muchas veces se enfoca en el sujeto que cursa por esta patología, dejando de lado a quienes tienen que vivirla, su red de apoyo o cuidadores principales, que necesitan tanto de un apoyo para seguir el proceso, como quienes la cursan; por lo cual se hace necesario ampliar los conocimientos a la disciplina de enfermería que brinden pautas de cuidado evitando así la pérdida de la autonomía y la sobrecarga del cuidador.(9) Es por esto que dicha teoría se articula con la investigación, ya que como se define anteriormente, la EM es una enfermedad crónica y degenerativa, que con el tiempo, conlleva a una discapacidad, generando una dependencia parcial, la cual después se convertirá en una dependencia total de su cuidador.(7).

La EM es actualmente la segunda causa de origen neurológico de limitación de la actividad en adultos jóvenes después de las lesiones traumáticas (encefálicas y espinales); ya que produce deterioro cognitivo en el $40-70 \%$ de todos las personas y afecta de modo variable las funciones cognitivas tales como la atención, la velocidad de procesamiento de la información, la memoria, la función ejecutiva y la percepción visual-espacial; se presentan comúnmente síntomas depresivos y de fatiga, lo que contribuye sustancialmente al impacto de la enfermedad en su calidad de vida diaria.(10)

La EM afecta a nivel personal y psicológico, se experimenta de manera diferente la enfermedad dependiendo de su situación en relación a las características individuales de cada caso, especialmente al comienzo de la enfermedad, aumenta el temor a empeorar, ese sentimiento se visualiza a través de la silla de ruedas y la falta de independencia o el miedo a la muerte, posiblemente debido a la falta de información en las primeras etapas. (11)

A nivel familiar social se produce una nueva forma de afrontar las relaciones familiares y de amistad, en donde se incluyen aspectos positivos y negativos; Entre los aspectos negativos, se produce pérdida de amistades y relaciones por la no comprensión de la adecuación a la nueva situación, que genera una modificación de actividades y pautas de conducta que se practicaban antes de la EM. En los aspectos positivos 
se involucra a la familia para amortiguar las consecuencias de la EM, y se aboga por una adaptación del individuo a las nuevas condiciones de vida. (5)

En el ámbito laboral y económico se presenta trauma por la ruptura con el trabajo; sin embargo, las consecuencias del cambio en la situación sociolaboral se pueden superar si se cuenta con la posibilidad de acceder a una pensión; el desempleo incrementa la dificultad de adaptación y aumenta la percepción de baja autoestima y autonomía. Por lo tanto, el trabajo no sólo suministra un soporte económico, sino un importante sentido de integración dentro de la comunidad.

(5)

En cuanto al personal de enfermería, al involucrarse en el proceso de enfermedad, no puede olvidar que la persona discapacitada espera confort y respeto a su dignidad y principios de autonomía, que en ocasiones sin intención de daño se omiten estos principios, acción que provoca el paternalismo, el cual se define como la limitación intencional de la autonomía de otra persona justificada por una apelación a la beneficencia, el bienestar o necesidades de otros; (8) no por ello se puede privar a la persona de su autonomía, ni del derecho a que se le trate con justicia y beneficencia. Es de vital importancia que todo el personal que tiene a su cargo la atención y cuidado de personas, profundice en el conocimiento de la Bioética y sus principios. (12)

Es decir la perspectiva de la enfermedad y la calidad de vida competen a las personas con EM, familiares y personales de salud que los atiende. Es por esto que se establece la siguiente pregunta ¿De qué manera afecta la esclerosis múltiple la calidad de vida, de la persona que la presenta, como a su red de apoyo y/o cuidador principal?

Por lo tanto se plantea como objetivo general de la investigación, realizar un análisis descriptivo de la esclerosis múltiple y la manera en que esta afecta la calidad de vida, tanto de quien la presenta, como a su red de apoyo y/o cuidador principal.

\section{METODOLOGÍA}

Se realizó una revisión bibliográfica la cual tiene como propósito presentar un análisis de la información más relevante sobre el tema de estudio; la elaboración de ésta pasa por tres grandes fases: la recolección de información, la cual se llevó a cabo a través de diferentes bases de datos (Medline, Scielo, Mediclatina, Medicasuis, ScholarAcademic, PubMed, Scopus, Fuente Académica Premier, Raco, Ebsco) con Descriptores de Ciencias de la Salud (DeCS) como: esclerosis múltiple, calidad de vida, enfermería, discapacidad, autoinmune. Se incluyeron artículos de investigación y revisión de tema que estuvieran dentro del rango de 2000 a 2015 y publicados en idioma castellano; se excluyeron artículos que estuvieran fuera del rango de años de búsqueda 2000-2015 y publicados en idiomas diferentes al castellano; seguido de la lectura y registro de la información mediante la elaboración de fichas bibliográficas en donde se contempló el fenómeno de estudio y finalizando con la redacción de un texto escrito.

Este estudio se apegó a lo declarado en la Resolución 8430 de Octubre 4 de 1993 dado que fue una investigación de bajo riesgo en donde no se empleó ningún tipo de intervención, asimismo prevaleció el respeto a la dignidad y la protección de los derechos y el bienestar de los seres humanos; por último contribuyó al conocimiento científico en enfermería (13).

\section{RESULTADOS}

Después de hacer una extensiva revisión en las bases de datos Medline, Scielo, Mediclatina, Medicasuis, ScholarAcademic, PubMed, Scopus, Fuente Académica Premier, Raco, Ebsco (2000 - 2015), se encontraron 50 artículos en revistas indexadas, de los cuales todos cumplieron con los criterios de inclusión a este estudio.

Analizando las diversas fuentes consultadas, en primer lugar se encontró que existen grandes similitudes en los resultados de 24 investigaciones ya que, es una enfermedad que afecta la calidad de vida de las personas en la misma manera, sin importar el lugar en el cual esta se desarrolle, teniendo en cuenta que las oportunidades para mejorar la calidad de vida de las personas después de un tratamiento, dependerá en gran medida del nivel de desarrollo en salud de la región en la que se presente el fenómeno.

Teniendo en cuenta que la EM presenta diferentes estadios de desarrollo y progresión, se encontraron diferentes estudios en los cuales:

Un estudio realizado en Cuba, mostró que el $80 \%$ de los pacientes con EM presento la forma clínica remisión-recaída (EMRR), un 7 \% primaria-progresiva (EMPP) y un $13 \%$ secundaria-progresiva (EMSP), (1) cifras que coinciden con otro estudio realizado en Donostia- España en el que participaron 114 pacientes (el 83,3\%, formas recurrentes remitentes o $\mathrm{RR}$; el 12,3\%, secundariamente progresivas o SP; el 
2,6\%, síndromes clínicamente aislados o CIS; el $0,9 \%$, primarias progresivas o $\mathrm{PP}$, y el $0,9 \%$, progresivas con recaídas o PR). 8

También se encontró que un alto porcentaje de las personas afectadas por EM tenían un nivel disminuido en cuanto a su independencia y empoderamiento con respecto a las diferentes actividades de su vida cotidiana. Un estudio realizado en Navarra - España, demostró que el 43,6\% de los encuestados necesito ayuda de otras personas para realizar alguna de las actividades básicas de la vida diaria, situación más frecuente entre la población femenina (un 49,5\%) que entre la masculina (el 29,3\%), lo cual conlleva a que requieran una asistencia continua por parte de sus familias y/o cuidadores, puesto que la gran mayoría (un $85,0 \%$ ) declara que necesita algún tipo de servicio de apoyo, especialmente más reclamado por la población femenina (un $89,9 \%$ frente al 73,2\% entre los varones). Entre quienes necesitan ayuda, las necesidades vitales más frecuentes están relacionadas con los desplazamientos fuera del hogar (un 52,5\%), con bañarse (un 39,4\%), con vestirse (un 29,5\%), o con levantarse /acostarse (un 27,8\%) (10), de igual manera, un estudio realizado en Madrid - España, mostró que más de la mitad de las personas afectadas por EM involucradas en dicho estudio requieren la ayuda de terceras personas en diversas situaciones de la vida cotidiana, ya sea ésta continuada o en momentos concretos.(16) Con respecto a la calidad de vida la Universidad de Murcia, España demostró en una investigación que la enfermedad afecta a la vida del sujeto de tal forma que sólo el 11,8\% reconoce no haber sufrido ningún cambio psicológico importante desde el momento de conocer el diagnóstico de la misma. Así, por ejemplo, el 70,6\% dice que sus relaciones sexuales han disminuido, y el 58,8\% de los sujetos reconoce un empeoramiento en la calidad de este tipo de contactos,(14) encontrándose relación con un estudio realizado en Noruega, el cual mostró que el 53\% de los pacientes con una puntuación de la discapacidad en Expanded Disability Status Scale (EDSS) $\leq 4$ presentaron problemas sexuales, lo cual conllevo a una reducción significativa de la calidad de vida (según el SF36), independientemente del estado de discapacidad (EDSS). Además, la disfunción sexual se relaciona con una menor vitalidad, presencia de dolor corporal, alteración de la función social y empeoramiento de la actividad mental. (15) Teniendo en cuenta que la existencia de un deterioro progresivo de la CVRS a lo largo de la evolución de la enfermedad puede llegar a valorarse como un estado peor que la muerte a partir de una puntuación de 8 en la EDSS mostrado en el estudio realizado en el Reino Unido. (18)

De la misma manera estudios sobre la calidad de vida relacionados con la salud (CVRS) realizados en Canadá, Noruega, España y Estados Unidos mostraron que el $70 \%$ de los pacientes se encontraban en situación de desempleo, y en el $50 \%$ de los casos debido a la propia EM. El $50 \%$ de los pacientes presentaron problemas en la ejecución de las tareas de la casa y desempleo a 10 años del inicio de la enfermedad, precisaron un apoyo para caminar tras 15 años y requirieron silla de ruedas 25 años después. Según el artículo Calidad de vida en esclerosis múltiple y otras enfermedades crónicas autoinmunes y no autoinmunes; los estudios realizados en Europa han mostrado que la fatiga asociada a la EM perturba notoriamente las funciones sociales y laborales de los pacientes, llegando incluso a ser la mayor causa de desempleo(15) datos que coinciden con un estudio realizado en Colombia, en donde se encontró que la fatiga es un síntoma común que ocurre hasta en el $97 \%$ de los pacientes con EM, Una tercera parte de los pacientes la consideran como su síntoma más incapacitante la cual puede ser un efecto directo de la EM o un efecto secundario del tratamiento.19 Asimismo, también se ha demostrado que la fatiga repercute significativamente en los dominios mentales y físicos de la CVRS según el SF36 , y su influencia en esta última es independiente de la puntuación de la EDSS.(15)

Tabla 1. Factores y componentes que afectan la calidad de vida.

Componente de

calidad de vida

Fatiga

Desempleo

Problemas en la ejecución de las

tareas de la casa

Porcentaje

Relaciones sexuales

$97 \%$
$70 \%-50 \%$

$50 \%$
$70,6 \%$
$43,6 \%$
$52,5 \%$
$39,4 \%$
$29,5 \%$
$27,8 \%$

El diagnostico precoz de la EM ayudan a detectar y realizar de manera temprana y oportuna un tratamiento. El diagnóstico preciso permite superar un período de incertidumbre y prepararse para enfrentar una enfermedad crónica con significativo impacto en muchos aspectos de la vida. 
Teniendo en cuenta lo anteriormente expuesto respecto a que la EM no solo afecta de forma física a la persona y a su entorno, se encontraron 14 estudios en donde exponen los diferentes tratamientos farmacológicos y no farmacológicos; en los farmacológicos se observa que si bien esta enfermedad no tiene cura, tiene un manejo para sus síntomas y en los no farmacológicos se encontró que existen fisioterapias y terapias alternativas las cuales ayudan a la persona a alcanzar y mantener las máximas capacidades físicas, psicológicas, sociales, vocacionales y una calidad de vida consistente; esta terapia se enfoca principalmente en aspectos de la propia persona, es decir que la dimensión emocional de la persona, que comprende su estado de ánimo, la manera de afrontar emocionalmente las circunstancias.

Por último se hallaron cinco estudios en donde resaltaron en sus resultados la importancia del cuidador y rol de enfermería en la EM; como se ha mencionado ya la persona con EM tiende a necesitar el apoyo y/o cuidado de una persona, esta generalmente es un familiar, que trata de satisfacer sus necesidades, llegando al punto de cambio en su vida cotidiana. Para esto se debe tener en cuenta que en el medio hospitalario el cuidado que brindan los enfermeros profesionales debe de tener como base la teoría del autocuidado de Dorothea Orem para así evitar que se vulnere la autonomía de la persona.

\section{DISCUSIÓN}

Relacionando los artículos obtenidos en la revisión sistemática se halló que la calidad de vida de las personas que presentan EM se afecta en todas las esferas y dimensiones humanas.

Se encontró un estudio sobre el concepto de calidad de vida, en el cual se está definiendo claramente en qué consiste este concepto como "la percepción del individuo sobre una situación en la vida dentro del contexto cultural y de los valores en la que vive, y en la relación con sus objetivos, normas e inquietudes" (4) mostrando las categorías que hacen parte de la calidad de vida; tales como:

1-Condiciones de vida, en la cual según O'Boyle (1994) plantea CV como la evaluación objetiva asume que la salud, ambiente físico, ingresos, vivienda y otros indicadores observables y cuantificables son mediciones válidas de $\mathrm{CV}$ y son estándares, sin embargo, la vinculación entre estos criterios objetivos $\mathrm{y}$ la CV percibida sería compleja y difícil de predecir.
2- Satisfacción con la vida en esta categoría el foco de interés es el bienestar subjetivo y dejar de lado las condiciones externas de vida, supone un individuo capaz de abstraerse, por ejemplo, de su contexto económico, social, cultural, político o incluso de su propio estado de salud, para lograr la satisfacción personal.

3-Condiciones de vida + Satisfacción con la vida desde esta perspectiva, las condiciones de vida pueden ser establecidas objetivamente mediante indicadores biológicos, sociales, materiales, conductuales y psicológicos, los que sumados a los sentimientos subjetivos sobre cada área pueden ser reflejados en el bienestar general.

4-Condiciones de vida + Satisfacción con la Vida * Valores esta es definida como un estado de bienestar general que comprende descriptores objetivos y evaluaciones subjetivas de bienestar físico, material, social y emocional, junto con el desarrollo personal y de actividades, todas estas mediadas por los valores personales.

5-Condiciones de vida + Satisfacción con la Vida * Evaluación percibido por un individuo estaría fuertemente modulado por los procesos cognitivos vinculados a la evaluación que la persona realiza tanto de sus condiciones objetivas de vida, como del grado de satisfacción con cada una de ellas; es por esto que se puede observar que el concepto de calidad de vida es complejo, y que por ende debe ser evaluado no sólo como un ítem de bienestar sino de forma holística.

Veinte y cuatro artículos relacionados directamente con la calidad de vida de las personas con esclerosis múltiple en los cuales se logra evidenciar que la calidad de vida es un fenómeno global biopsicosocial. La EM deteriora de manera progresiva diferentes funciones neurológicas, que repercuten en el sistema musculo esquelético; esto determina una disminución en la calidad de vida, principalmente en las funciones física y social.(16) la EM se caracteriza porque la persona cruza por diferentes etapas con sentimientos muy diversos, sin contar con el hecho que el curso de la enfermedad es diferente en cada persona, suele aparece un sentimiento común en todos los casos, especialmente al comienzo de la enfermedad, el temor a empeorar; ese sentimiento se visualiza a través de la silla de ruedas y la falta de independencia o el miedo a la muerte, posiblemente debido a la falta de información en las primeras etapas. frente a este sentimiento las personas utilizan como estrategia no tener contacto con otras personas afectadas ni saber mucho sobre la enfermedad, intentando pensar que no les está pasando y mantener la normalidad en sus vidas.(11) 
con respecto a las alteraciones emocionales que se presentan principalmente son la ansiedad y depresión. (17) las personas indican una peor calidad de vida relacionada con la salud fuertemente relacionada con la depresión y ansiedad,(13) estas, además del sueño, se relacionaron con varios componentes de la misma como: las limitaciones del rol por problemas físicos y emocionales, dolor, energía, función social, función cognitiva, función sexual y cambio en la salud;(1) Los niveles de depresión son mayores en los pacientes con alteraciones cognitivas en atención y velocidad de procesamiento y en memoria en comparación con los pacientes que mantienen preservadas estas funciones,(18) además, se ve reflejado el enlentecimiento en el procesamiento de la información y dificultades atencionales y el deterioro cognitivo es un fenómeno frecuente que depende casi exclusivamente del tiempo de evolución, y es mayor en pacientes con formas progresivas, lo que le otorga valor pronóstico por lo cual estamos obligados a evaluar y tratar el deterioro cognitivo en la $\operatorname{EM}(1)$, sin embargo, hay que tener en cuenta que este tipo de pacientes puede mejorar la calidad de vida propia y de su familia, estimulando al enfermo a adoptar estrategias de afrontamiento adecuadas que disminuyan el estrés y la posibilidad de deterioro cognitivo, aydándole asumir suenfermedad, y dando pautas a la familia para que no se sobreproteja al paciente.(5) Por lo tanto se ha demostrado que la calidad de vida muestra una mayor relación con la adaptación a la enfermedad que con sus síntomas,(10) siendo de gran importancia su estudio. Catorce estudios sobre los diferentes tratamientos tanto farmacológicos como fisioterapéuticos y de terapias alternativas, en los cuales se muestra formas de aportar y tratar de potenciar la calidad de vida de la persona con EM ya que es muy discapacitante; por un lado se tiene los tratamientos farmacológicos, estos se enfocan primordialmente en la analgesia, ya que una de las principales afectaciones de la calidad de vida es el dolor; entonces en diferentes estudios se encuentra que los fármaco más utilizado es el cannabinoide el cual tiene efectos secundarios son leves, siendo los más frecuentes vértigo, xerostomía, somnolencia y alteraciones en el equilibrio los cuales no alteran la calidad de vida del paciente,(19) también se administran fármacos como la Morfina, Lidocaína, Mexiletina, combinación de lofepramina Vitamina B12, L-fenilalanina,(20) pero estos últimos son menos usados porque tienen efectos adversos más fuertes y son más costosos para el Estado; en pocas palabras los tratamientos que se vayan a utilizar en estas personas debe ser con gran eficacia y efectividad y con menos efectos adversos, que evite el abandono de los tratamientos por parte de las personas con EM garantizando mejor calidad de vida y desempeño de sus actividades de la vida cotidiana,(19) por otro lado están las fisioterapias y las terapias alternativas, en primer lugar está el uso de terapias precoces como la neurorrehabilitación en la EM,(20) proceso que ayuda a la persona a alcanzar y mantener las máximas capacidades físicas, psicológicas, sociales, vocacionales y una calidad de vida consistente. Es por esto que la neurorrehabilitación debe ser considerada en todas las etapas de la enfermedad;(21) en segundo lugar está la psicología positiva la cual busca la mejora de los aspectos positivos que posee la persona para poder hacer frente a su propia vida de la mejor forma posible, teniendo como meta conseguir una buena calidad de vida,(22) esta terapia se enfoca principalmente en aspectos de la propia persona, es decir que la dimensión emocional de la persona, que comprende su estado de ánimo, la manera de afrontar emocionalmente las circunstancias que van aconteciendo en su vida, la repercusión de la presencia o ausencia de vínculos interpersonales con familiares y amigos, los recursos psicológicos para gestionar su enfermedad,(23) todos estos factores influyen en la calidad de vida de la persona con EM, es por esto, que esta terapia es ideal para potenciar aspectos psicológicos y así mejorar la calidad de vida.

Cinco investigaciones acerca del cuidado que desempeña enfermería y los cuidadores principales, los cuales presentan una estrecha relación con la teoría del déficit de autocuidado y la calidad de vida dado que se halla los roles que desempeñan las personas con EM y el enfermero o cuidador principal; en primer lugar están las personas con EM las cuales dependiendo del grado de la enfermedad pueden presentar limitaciones en la satisfacción de sus necesidades de cuidado, presentando así un déficit de autocuidado, situando así en segundo lugar la acción que desempeñan los enfermeros, enfermeras o cuidadores principales, aquellos son de vital importancia en el curso de la enfermedad ya que ejercen varios papeles; aquí tenemos el uso de los sistemas enfermeros utilizados de forma directa por los profesionales de enfermería,(24) los cuales en el medio hospitalario son los que suplen las actividades de autocuidado empleando el primer sistema, el que es el totalmente compensador, el cual consiste en cumplir con el autocuidado de la persona, compensar la incapacidad de la persona de comprometerse y desarrollar un autocuidado, da apoyo a la persona y le 
protege, este sistema es muy útil para esta enfermedad en los estadios más avanzados porque genera discapacidad, además de que necesitan sostén en su parte psicológica y emocional pero a la vez surge la pérdida de la autonomía y está el parcialmente compensador, este desarrolla algunas medidas de autocuidado para la persona siendo este el que desempeña parcialmente su autocuidado; regula la actividad de autocuidado; acepta el cuidado y ayuda a la enfermera o enfermero, cuando la persona con EM no se encuentra en un estadío avanzado, y la utilización de estos sistemas de forma indirecta es por parte de los cuidadores principales, en donde pueden llegar al sindrome de carga del cuidador,(25) porque el estado de ánimo se ve afectado presentado sentimiento de culpa e irritabilidad, también influyendo en las labores de la vida cotidiana como incapacidad para trabajar, trastornos de sueño, fatiga y pérdida del apetito, es por esto que enfermería debe manejar el último sistema con el nombre de apoyo educativo hacia los cuidadores principales para que no presenten este síndrome ya que se le pueden brindar pautas para cuidado de las personas con EM desde un enfoque en donde beneficie tanto a la persona como a su cuidador y así regulando el ejercicio y desarrollo de la actividad de autocuidado; planteados por Orem en su teoría. Todos los sistemas empleados, ya sea por parte de enfermería o cuidadores principales van encaminados a la mejora de la calidad de vida de la persona.

Cinco estudios sobre la prevalencia de la EM en Colombia y a nivel internacional, determinaron que la prevalencia de EM en Colombia aumentó, y que el país podría encontrarse en riesgo intermedio, con una prevalencia nacional de 7,52 por cada 100.000 , al comparar esta prevalencia con la de otros países latinoamericanos, se encuentra que naciones como Argentina, Brasil, Chile y Cuba reportan mayores prevalencias que la nuestra, mientras que Perú tiene una prevalencia similar a Colombia, y tanto Ecuador como México cuentan con las más bajas de la región, teniendo en Colombia mayor prevalencia en Bogotá,(26) además se correlacionan ya que estos planean que la prevalencia aumenta con la latitud, es poco común en las zonas tropicales y afecta más a la raza blanca y al sexo femenino.(27)

Una investigación encontrada valora los criterios Mc Donald 2010, los cuales ayudan a detectar y realizar de manera temprana y precoz el diagnóstico de EM, este es de gran importancia ya que el diagnóstico de la EM ha sido una verdadera preocupación para los pacientes y para los médicos, para los primeros, porque el diagnóstico preciso permite superar un período de incertidumbre y prepararse para enfrentar una enfermedad crónica con significativo impacto en muchos aspectos de la vida, y para los segundos porque la ausencia de una prueba diagnóstica específica suele resultar en un proceso de búsqueda de exámenes a favor o en contra de la enfermedad. (28)

Esta ausencia de un test diagnóstico específico, en el pasado dificultó realizar un diagnóstico con certeza, permitiendo que dicha duda generara errores con sobrecostos en los sistemas de salud al tratar pacientes que no padecían la enfermedad. (28) La investigación realizada en Cali (2010) sobre los criterios Mc Donald busca concientizar a los profesionales de salud para la aplicación correcta de dichos criterios ya que es una enfermedad catastrófica, por eso se busca una buena y completa historia clínica, así como la revisión de las imágenes, esto permitirá su identificación temprana con el fin de llevar a cabo intervenciones oportunas que conduzcan a un tratamiento adecuado, disminuyendo las tasas de recaídas y por ende de discapacidad, lo que llevará a una mejor calidad de vida. (28)

Teniendo en cuenta lo anterior, se puede concluir que la calidad de vida de las personas que padecen esclerosis múltiple se ve afectada de manera drástica ya que es una enfermedad que puede llegar a ser complemente discapacitante en estadios avanzados si no se lleva a cabo un tratamiento oportuno que busque mejorar la calidad de vida de la persona, lo cual repercute directamente a nivel personal, familiar, emocional, psicológico, laboral y social, viéndose afectada no solo la persona que la presenta sino también, su red de apoyo o cuidadores, debido a la sobrecarga que estos pueden llegar a presentar, lo cual puede generar niveles elevados de ansiedad, estrés y depresión que repercuten significativamente en la salud física y mental de la persona, con el riesgo de producir aislamiento social, falta de tiempo libre tanto personal como familiar, deterioro de la situación económica y laboral, etc.; alterando de esta manera el bienestar integral de la persona, por tal motivo, es de vital importancia que desde la disciplina de enfermería se realicen intervenciones a nivel familiar, buscando mejorar la calidad devida delapersona con EM y de su red de apoyo y/o cuidadores, con el fin de disminuir las consecuencias negativas en el bienestar tanto a nivel personal como familiar, dichas intervenciones se pueden abordar desde el ámbito educativo, ya que una adecuada y oportuna educación a las personas involucra- 
das directamente (persona diagnosticada con EM y familia/cuidador) puede llegar a generar una mayor resiliencia, lo cual permitirá que el afrontamiento a las situaciones de estrés generado por el proceso de enfermedad sea mucho más fácil de llevar, de esta manera se puede contribuir a que el impacto en la calidad de vida de las personas con EM y su familia, se vea significativamente disminuido.

Por otra parte, se pudo evidenciar que no se encuentran suficientes artículos que midan la calidad de vida de las personas diagnosticadas con esta enfermedad, ni la de sus familiares, mostrando así la importancia de realizar más investigaciones que contribuyan con el manejo y atención de estas personas, reconociendo la importancia de que al brindar cuidado de manera multidisciplinaria y holística, se podrían disminuir costos al SGSSS, al gobierno y a los mismos familiares y/o cuidadores que son los que directamente se involucran en el cuidado de la persona yquienes de igual manera necesitan ser tomados en cuenta y quese les brinde una atención integral para evitar consecuencias negativas a nivel físico, emocional, psicológico, económico,laboral, entre otras; que de una u otra manera generará más gastos al estado en general.

Por tal motivo es importante que se generen estrategias desde las entidades gubernamentales y prestadoras de salud, las cuales permitan llevar a cabo oportunidades para mejorar la calidad de vida de las personas, además de fortalecer el desarrollo de investigaciones futuras en nuestras región, con el fin de ampliar el panorama de la EM en Colombia ya que se encontraron pocos estudios y leyes que fundamentan el estado de esta enfermedad, con el fin de adquirir mayor capacidad de brindar cuidados integrales que se adecuen a las necesidad es de las personas de la región.

\section{BIBLIOGRAFÍA}

- 1. Companioni I, Jiménez R Morales, Jiménez, Nápoles Y, Macías Y. Calidad de vida en la esclerosis múltiple: su elación con la depresión, fatiga y calidad del sueño. Rev. Scielo [revista en línea]. 2013. [citado: 07 abril 2016]. Disponible http://scielo.sld.cu/pdf/gme/v15n3/gme03313.pdf

- 2. Sánchez J, Aguirre C, Arcos O, Jiménez I, Jiménez M, León F, Pareja J, Pradilla G, B Uribe, Uribe C, villa A, Volcy M, Palacio L. Prevalencia de la esclerosis múltiple en Colombia. Rev. Neurol [revista en línea]. 2000 [citado: 8 abril 2016]. Disponible en: http://www.neurologia.com/pdf/web/3112/ j121101.pdf

- 3. Vizcarra D, Kawano J, Castañeda C, Chereque A, Tipismana M, Bernabé A, Juarez A. Prevalencia de la esclerosis múltiple en Lima-Perú. Rev. MedHered [revista en línea]. 2009 [citado: 8 abril 2016]. Disponible en: http://www.scielo.org.pe/scielo.php?script=sci_artt

- 4. Urzúa A,Caqueo A. Calidad de vida: Una revisión teórica del concepto. Revscielo [revista en línea]. 2012 [citado: 8 abril 2016]. Disponible en: http://www.scielo.cl/scielo.php?pid=S071848082012000100006\&amp;script=sci_arttext

- 5. Rivera J, Morales J, León J, Mitchell A. Dimensión social y familiar: experiencias de cuidadores y personas con esclerosis múltiple. El estudio gedma. Rev. Neurol. 2008 [citado: 15 abril 2016]. Disponible en: http://gredos.usal.es/xmlui/bitstream/handle/10366/22610/DSC_DimensionSocialFamiliar. pdf.pdf?sequence $=1$

- 6. Romero M, Arango C, Alvis N, Suárez J C, Duque A, MD, Costos de la Esclerosis Múltiple en Colombia. Rev. Neurol [revista en línea]. 2011. [citado: 21 abril 2016]. Disponible en: https://www. ispor.org/consortiums/LatinAmerica/documents/ViH/Costos-de-la-Esclerosis-Multiple.pdf

- 7. Alligood M, Marriner A. Modelos y teorías en enfermería, séptima edición. Barcelona: Elsevier; 2011 [citado: 27 abril 2016]

- 8. González Y, Luna J. Enfermería y bioética en pacientes con esclerosis múltiple, ingresados en el Hospital de Rehabilitación. 2004-2006 Rev. Cubana Enfermer 2007 [citado: 8 abril 2016]. Disponible en: http://scielo.sld.cu/scielo.php?pid=S0864-03192007000200007\&script=sci_arttext\&tlng=pt

- 9. López M, Orueta R, Gómez S, Sánchez, Carmona J, Alonso F. El rol de Cuidador de personas dependientes y sus repercusiones sobre su Calidad de Vida y su Salud. Rev. ClinMed Fam. 2009 [citado: 11 mayo 2016] disponible en: http://www.revclinmedfam.com/PDFs/32bb90e8976aab5298d5da10fe6 6f21d.pdf

- 10. Sistiaga A, Castillo T, Aliri J, Gaztañaga i, Acha J, Arruti M, Otaegui D, Olascoaga J. Rendimiento cognitivo y calidad de vida de la esclerosis múltiple en Gipuzkoa. Rev. neurol [revista en línea]. 2014 [citado: 7 abril 2016]; 58 (8). Disponible en: Carrón J, Arza J. Esclerosis múltiple: análisis de 
- necesidades y calidad de vida de los afectados y su entorno. España. Rev. Española de Discapacidad. 2013. [citado 9 de abril de 2016] disponible en : http://www.cedd.net/redis/index.php/redis/article/ view/65/57

- 11. Arbinaga F. Aspectos emocionales y calidad de vida en pacientes con enfermedades desmielinizantes: el caso de la esclerosis múltiple. Rev. Anales de Psicología [revista en línea] 2013. [citado: 07 abril 2016]. Disponible en: http://www.discapacidadonline.com/wp-content/uploads/2012/07/ aspectos-emocioales-calidad-de-vida-esclerosis-multipple.pdf

- 12. Resolución $N^{\circ} 008430$ De 1993, de Investigación en Salud. (4 de Octubre de 1993) [citado 29 de abril de 2016] disponible en: https://www.unisabana.edu.co/fileadmin/Documentos/Investigacion/ comite_de_etica/Res_8430_1993_-_Salud.pdf

- 13. Farfán J, Espitia $\mathrm{O}, \mathrm{MD}$. Esclerosis múltiple en pacientes pediátricos: fisiopatología, diagnóstico y manejo. Colombia Rev. MedUnab. 2012 [citado 9 de abril de 2016] disponible en: http://132.248.9.34/ hevila/Medunab/2011-12/vol14/no3/4.pdf

- 14. Restrepo D, Calle J. Aspectos neuropsiquiátricos de la enfermedad de Wilson y la esclerosis múltiple. Rev. Scielo [revista en línea]. 2007 [citado 11 de abril de 2016] disponible en: http://www.scielo. org.co/pdf/rcp/v36s1/v36s1a11.pdf

- 15. Hincapié M.E, Suárez J.C, Pineda R, Anaya J.M. . Calidad de vida en esclerosis múltiple y otras enfermedades crónicas autoinmunes y no autoinmunes. Rev. Neurol [revista en línea]. 2009. [citado: 9 abril 2016]; 48(5) disponible en:http://www.coloquiodiscapacidad.com/investigaciones/phocadownload/Salud-vida-y-funcionamiento-humano/hincapie_otros_2009.pdf

- 16. Godoy J F, Muela J, Pérez M. Aspectos emocionales de la esclerosis múltiple. Rev canales de psicología 1993 [citado: 8 abril 2016]. Disponible en: http://revistas.um.es/analesps/article/viewFile/29221/28281

- 17. Nieto A, Sánchez M, Barroso J, Olivares T, Hernández M. Alteraciones cognitivas en etapas iniciales de la esclerosis múltiple y su relación con el estado de ánimo, variables demográficas y clínicas. Psicothema [revista en línea] 2008. Disponible en: http://eds.a.ebscohost.com.uniquindio.elogim. com/eds/pdfviewer/pdfviewer?sid=9dbf5753-2198-42bc-9b79-0d6914fb210e\%40sessionmgr4001\&v id $=31 \&$ hid $=4203$

- 18. Maldonado J. M.D, Álvarez A. M.D, Rodríguez M. M.D. Efectividad Y Efectos Secundarios Del Tratamiento Con Canabinoides En Dolor Neuropático De Tipo Central En Pacientes Con Esclerosis Múltiple Rev. Med. [revista en línea]. 2010 [citado 18 de mayo de 2016] disponible en : http://www. scielo.org.co/pdf/med/v18n1/v18n1a08.pdf

- 19. Correa D., Moreno C. Revisión sistemática de la literatura:tratamiento del dolor central en esclerosis múltiple Rev. Neurol.[revista en línea] 2009. citado 18 de mayo de 2016] disponible en : http:// www.acnweb.org/acta/2009_25_1_4.pdf

- 20. Ayán C., de Paz J., Pérez D. Esclerosis múltiple y ejercicio físico: revisión bibliográfica, situación actual y perspectivas de futuro. Rev. Medicina Esport. [revista en línea] 2004 [citado 18 de mayo de 2016] disponible en : http://apps.elsevier.es/watermark/ctl_servlet?_f=10\&amp;pident articulo $=13105974 \& a m p ; p i d e n t \_u s u a r i o=0 \& a m p ; p$ contactid $=\& a m p ; p i d e n t$ revista $=277 \& a m p ; t y=2 \overline{1}$ \&amp;accion=L\&amp;origen=bronco\%20\&amp;web=www.apunts.org\&amp;lan=es\&amp;fichero=2 77v39n144a13105974pdf001.pdf

- 21. Real Y., López M., Díaz R., Cabrera J. Efectividad de un programa de rehabilitación respiratoria en pacientes con esclerosis múltiple Rev. Cubana de Salud publica [revista en línea]. 2012 [citado 18 de mayo de 2016] disponible en : http://scielo.sld.cu/pdf/rcsp/v37n1/spu03111.pdf

- 22. Belén A., Fajardo M. Eficacia de un programa de psicología positiva en un adolescente con esclerosis múltiple. Revista de Psicología, No1-Vol.1 [revista en línea]. 2014 [citado: 18 mayo 2016]. Disponible en:http://dehesa.unex.es/xmlui/bitstream/handle/10662/2058/0214-9877_2014_1_1_379. pdf? sequence $=4$

- 23. Robles M., Merchan M., Guerrero J., Cruz V. El rol de enfermería en la mejora de la calidad de vida del paciente con esclerosis múltiple. Rev. CientSocEspEnfermNeurol. 2015 [citado: 8 abril 2016]. Disponible en: http://apps.elsevier.es/watermark/ctl_servlet?_f=10\&amp;pident articulo $=90444238 \&$ amp;pident $\_$usuario $=0 \&$ amp;pcontactid $=\& a m p ;$ pident_revista $=319 \& a m p ; t y=1 \overline{1}$ $5 \&$ amp;accion=L\&amp;origen=zonadelectura\&amp;web=www.elsevier.es\&amp;lan=es\&amp;ficher $\mathrm{o}=319 \mathrm{v} 42 \mathrm{n} 01 \mathrm{a} 90444238 \mathrm{pdf001.pdf}$ 
- 24. Macías Y, Pedraza H, Jiménez R, Pérez M, Valle R, Fundara R. Sobrecarga en los cuidadores primarios de pacientes con esclerosis múltiple: Su relación con la depresión y ansiedad. Revista Mexicana de Neurociencia [Revista en línea] 2014. [citado: 8 abril 2016].Disponible en:http://eds.a.ebscohost. com.uniquindio.elogim.com/eds/pdfviewer/pdfviewer?sid=429be085-7f53-42b0-9f29-6e3d75a4a52d \%40sessionmgr4003\&amp; vid=30\&amp;hid=4105

- 25. Cheryl E. Jiménez-Pérez, Luis A. Zarco-Montero, Camilo Castañeda-Cardona, Margarita Otálora Esteban, Andrea Martínez, Diego Rosselli Estado actual de la esclerosis múltiple en Colombia Rev. scielo [revista en línea]. 2015 [citado 9 de abril de 2016] disponible en : http://www.scielo.org.co/pdf/ anco/v31n4/v31n4a05.pdf

- 26. Bermejo P., Oreja C., Díez E. El dolor en la esclerosis múltiple: prevalencia, mecanismos, tipos y tratamiento. Rev. Neurología [revista en línea]. 2010 [citado 11 de abril de 2016] disponible en : http:// www.neurologia.com/pdf/Web/5002/bd020101.pdf

- 27. Diazgranados J., Burbano J., Herrera A., Hidalgo J., Gómez L., Guevara L. Criterios Mc Donald 2010 en el diagnóstico de esclerosis múltiple en Cali, Colombia. RevScielo 2010 [citado: 8 abril 2016]. Disponible en: http://www.scielo.org.co/pdf/anco/v29n4/v29n4a04.pdf

- $\quad$ 28. Pareja J., Torres M., Castillo E., Covo P., Berrocal M., Polo L., Zequeira R., González J., Guzmán D., Alwers R. Discapacidad física en la práctica clínica diaria en pacientes con esclerosis múltiple tratados con interferón beta-1b Colombia Rev. scielo [revista en línea]. 2013 [citado 9 de abril de 2016] disponible en: http://www.scielo.org.co/pdf/anco/v29n2/v29n2a04.pdf

- 29. Pérez M. Afrontando la esclerosis múltiple: sentimientos y temores. RevPortularia [revista en línea]. 2012 [citado: 8 abril 2016] Vol. XII, N². Disponible en: http://rabida.uhu.es/dspace/bitstream/ handle/10272/6240/Afrontando_la_esclerosis.pdf?sequence $=2$

- 30. Ley 266 de 1996, Reglamentación de la profesion de enfermeria en Colombia.(Diario Oficial No. 42.710, del 5 de febrero de 1996) [citado 9 de abril de 2016] Disponible en: http://www.mineducacion. gov.co/1759/articles-105002_archivo_pdf.pdf

- 31. Olascoaga J. Calidad de vida y esclerosis múltiple. Rev. Neurol [revista en línea] 2010.[citado 9 de abril de 2016] disponible en: https://es.scribd.com/doc/38271429/Calidad-de- Vida-en-La-Esclerosis- Multiple

- $\quad$ 32. Nogales J., Aracena R., Cepeda S., Eloiza C., Agurto P., Díaz V., Labbé S., Martínez S., Flores J., Araya C.. Esclerosis múltiple recurrente remitente en el sector público de salud de Chile. Descripción clínica de 314 pacientes Rev. scielo [revista en línea]. 2014 [citado 9 de abril de 2016]disponible en: http://www.scielo.cl/pdf/rmc/v142n5/art02.pdf

- 33. Nogales J., Aracena, Díaz V., Zitko P., Eloiza C., Cepeda S., Agurto P., González C. Evaluación neuropsicológica en 129 pacientes chilenos con esclerosis múltiple recurrente remitente previo al inicio de fármacos inmunomoduladores Rev. Scielo [revista en línea]. 2012 [citado 9 de abril de 2016] disponible en: http://www.scielo.cl/pdf/rmc/v140n11/art09.pdf

- 34. Salinas V., Rogero P., Oña A., Vergara M. Descubriendo el significado de los fenómenos cronicidad, progresión y singularidad en el diagnóstico de la esclerosis múltiple Revscielo [revista en línea]. 2012 [citado 9 de abril de 2016] disponible en: http://scielo.isciii.es/scielo.php?script=sci arttext\&amp;pid=S1132-12962012000200004\&amp;lng=pt\&amp;tlng=es

- 35. Mestas L., Salvador J., Gordillo F. Reserva cognitiva y déficit en la planificación en pacientes con esclerosis múltiple. Rev. scielo [revista en línea]. 2012 [citado 9 de abril de 2011] disponible en: http:// scielo.isciii.es/pdf/neuropsiq/v32n113/original4.pdf

- 36. Pino M., Olivares T., Nieto A., Hernández J., Barroso J.Esclerosis múltiple y depresión. II Congreso Internacional de Neuropsicología en Internet 2003 [citado: 8 abril 2016]. Disponible en: http://sid. usal.es/idocs/F8/ART9783/esclerosis_multiple_depresion.pdf

- Paes R., Alvarenga R., Vasconcelos C., Negreiros M., Landeira M. Neuropsicología de la esclerosis múltiple primaria progresiva REV NEUROL 2009 [citado: 8 abril 2016]. Disponible en: http://www. neurologia.com/pdf/web/4907/bc070343.pdf

- 38. Velázquez E, Valero A, Cruz N. Condiciones de vida de pacientes de 15 a 29 años con diagnóstico de esclerosis múltiple. Archivos de Neurociencias.[Artículo en línea] 2007. Disponible en:http:// eds.a.ebscohost.com.uniquindio.elogim.com/eds/pdfviewer/pdfviewer?sid=429be085-7f53-42b09f29-6e3d75a4a52d\%40sessionmgr4003\&amp;vid=7\&amp;hid=4105 
- Magaña L, Chiquete E, Campos I, Cantú R, Ibarra O, Punzo G, Rojas I. Factores de riesgo y pronóstico de pacientes con esclerosis múltiple del estado de Michoacán, México: Un estudio de casos y controles. Revista Mexicana de Neurociencia [revista en línea] 2012. Disponible en:

- $\quad$ http://eds.a.ebscohost.com.uniquindio.elogim.com/eds/pdfviewer/pdfviewer?sid=9dbf57532198-42bc-9b79-0d6914fb210e\%40sessionmgr4001\&amp;vid=18\&amp;hid=4203

- 40. Jiménez J, Kleinert A, Rodríguez D, Molina L. Impacto cualitativo de fallas cognitivas sobre las actividades de la vida diaria en pacientes con esclerosis múltiple de reciente diagnóstico. Archivos de Neurociencias. [Revista en línea] 2012. Disponible en:http://eds.a.ebscohost.com.uniquindio.elogim. com/eds/pdfviewer/pdfviewer?sid=9dbf5753-2198-42bc-9b79-0d6914fb210e\%40sessionmgr4001\&a $\mathrm{mp} ;$ vid=45\&amp;hid $=420$

- 41. Vinaccia S, Quiceno J. Calidad de vida relacionada con la salud y enfermedad crónica: estudios colombianos. Editorial bonaventuriana [artículo en línea] 2012.Disponible en: http://www.revistas. usb.edu.co/index.php/Psychologia/article/view/1175/9

- 42. Andrés C, Guillem A. Una aproximación sobre la calidad de vida en pacientes con esclerosis múltiple. Revista neurol [Revista en línea] 2000. Disponible en: http://saudepublica.bvs.br/pesquisa/ resource/pt/ibc $\neg 20534$

- 43. Rodríguez Al. Estudio de casos y controles sobre la influencia del hábito tabáquico en la esclerosis múltiple.Neurología. Pontevedra. 2009 [citado 9 de abril de 2016] disponible en: http://sid.usal.es/ idocs/F8/ART13170/estudio_de_casos_y_controles.pdf

- 44. Rosselli D., Ariza J., Acosta T., Rueda J. Análisis de impacto presupuestal de la introducción de fingolimod en el tratamiento de esclerosis múltiple en Colombia.Colombia. Rev. Scielo, 2012.[citado 9 de abril de 2016] disponible en: http://www.scielo.org.co/pdf/anco/v28n1/v28n1a02.pdf

- 45. Terré $\neg$ Boliart R., López F. Tratamiento rehabilitador Rev. Neurol [revista en línea]. 2007 [acceso 11 de abril de 2016];44(7): 426 431 disponible en: http://www.neurologia.com/pdf/web/4407/ x070426.pdf

- 46. Arnett P., Forn C. Evaluación neuropsicológica en la esclerosis múltiple Rev. Neurología [revista en línea].2007 [citado 11 de abril de 2016] disponible en: http://www.neurologia.com/pdf/Web/4403/ x030166.pdf

- 47. Martínez N., Hernández R., y Hernández B. Estimulación a las áreas de lenguaje, memoria y motricidad en personas adultas afectadas por la esclerosis múltiple. [Revista en línea]. [citado 11 de abril de 2011] disponible en: http://200.23.113.50:8080/upn/bitstream/handle/11195/399/ Estimulaci\%C3\%B3n\%20a\%20las\%20\%C3\%A1 reas\%20del\%20lenguaje \%2c\%20memoria\%20 y\%20motricidad\%20en\%20personas\%20adultas.pdf?sequence $=1$

- 48. Arza J., Carrón J. Esclerosis Múltiple, Calidad de vida y Atención Sociosanitaria. Rev. contrapunto [revista en línea]. 2011 [citado: 7 abril 2016]. Disponible en: http://www3.uah.es/congresoreps2013/Paneles/panel4/sesion1/javier.arza@unavarra.es/TCArza.pdf

- 49. Delgado $\neg$ J., Cadenas $\neg$ J. , Fernández J., Navarro G., Izquierdo G. Estudio de la calidad de vida en la esclerosis múltiple. Rev. Neurol [revista en linea]. 2005. [citado: 8 abril 2016]; 41(5). Disponible en: http://www.neurologia.com/pdf/Web/4105/t050257.pdf

- 50. Jiménez R.,DíazS.,Luna J., Brunet D., Osmani A., Dorta -A. Experiencia de un Programa de Neurorrehabilitación Integral en adultos con esclerosis múltiple en Cuba. Revista Mexicana de Medicina Física y Rehabilitación [revista en linea]. 2009. [citado: 9 abril 2016]; 21(4).Disponible en: http:// www.medigraphic.com/pdfs/fisica/mf-2009/mf094c.pdf

- 51. Álvarez S., Quiñones S., Plascencia N., Sauri S., Núñez L. Impacto de la disfunción sexual en la calidad de vida en pacientes con esclerosis múltiple. Rev. Mexneuroci [revista en línea]. 2007 [citado: 9 abril 2016]; 8(2). Disponible en: http://revmexneuroci.com/wp-content/uploads/2014/06/Nm072 $\neg 07$. pdf

- 52. Sistiaga A, Castillo T, Aliri J, Gaztañaga i, Acha J, Arruti M, Otaegui D, Olascoaga J. Rendimiento cognitivo y calidad de vida de la esclerosis múltiple en Gipuzkoa. Rev. neurol [revista en línea]. 2014 [citado: 7 abril 2016]; 58 (8). Disponible en: http://www.neurologia.com/pdf/Web/5808/ b1080337.pdf 